\title{
EVENTO CONTEXTUALIZADO EN INGENIERÍA: TAREAS DOCENTES Y CONOCIMIENTOS MOVILIZADOS EN ELLAS
}

\author{
Gabriel Loureiro de Lima \\ gloureirolima@gmail.com \\ Pontifícia Universidade Católica de São Paulo (PUC-SP) \\ https://orcid.org/0000-0002-5723-0582
}

Eloiza Gomes

eloiza@maua.br

Instituto Mauá de Tecnologia - IMT

https://orcid.org/0000-0002-1217-9904

Barbara Lutaif Bianchini

barbaralb@gmail.com

Pontifícia Universidade Católica de São Paulo (PUC-SP)

https://orcid.org/0000-0003-0388-1985

Recibido: 15/12/2020 Aceptado: 14 de mayo de 2021

\section{Resumen}

El objetivo de este trabajo, de naturaleza teórico-bibliográfica, es reflexionar acerca de la movilización de categorías de conocimientos docentes esenciales para ejecutar tareas inherentes al Modelo Didáctico de la Matemática en Contexto, relacionado a la Teoría de La Matemática en el Contexto de las Ciencias. A partir de un estudio sobre categorías de conocimientos docentes, desde el punto de vista de diversos investigadores, identificamos las que tienen que ser accionadas en las ocho tareas que el profesor desempeña cuando construye un evento contextualizado, señalando algunas a partir de un evento construido para una disciplina inicial de Cálculo Diferencial e Integral en un curso de Ingeniería Civil. Por medio del análisis realizado, concluimos que la mayor parte de las tareas requiere la movilización, siempre de manera simultánea y articulada, de conocimientos de contenido, didácticos, pedagógicos y tecnológicos.

Palabras Clave: Categorías de conocimientos docentes; Modelo Didáctico de la Matemática en el Contexto de las Ciencias; Matemática; Ingeniería; Evento contextualizado.

\section{EVENTO CONTEXTUALIZADO NA ENGENHARIA: TAREFAS E CONHECIMENTOS DOCENTES NELAS MOBILIZADOS}

\begin{abstract}
Resumo
O objetivo deste trabalho, de natureza teórico-bibliográfica, é refletir acerca da mobilização de categorias de conhecimentos docentes essenciais para a execução de tarefas inerentes ao Modelo Didático da Matemática em Contexto, atrelado à Teoria A Matemática no Contexto das Ciências. A partir de um estudo sobre categorias de conhecimentos docentes, na visão de diversos pesquisadores, identificamos aquelas que precisam ser postas em ação nas oito tarefas que o professor desempenha quando constrói um evento contextualizado, apontando algumas delas a partir de um evento construído para uma disciplina inicial de Cálculo Diferencial e Integral em um curso de Engenharia Civil. Por meio da análise realizada, concluímos que a maior parte das
\end{abstract}


tarefas requer a mobilização, sempre de maneira simultânea e articulada, de conhecimentos de conteúdo, didáticos, pedagógicos e tecnológicos.

Palavras-Chave: Categorias de conhecimentos docentes; Modelo Didático da Matemática em Contexto das Ciências; Matemática; Engenharia; Evento contextualizado.

\title{
CONTEXTUALIZED EVENT IN ENGINEERING: TEACHING TASKS AND KNOWLEDGE MOBILIZED IN THEM
}

\begin{abstract}
The objective of this paper, of a theoretical-bibliographic nature, is to reflect on the mobilization of categories of essential teaching knowledge for the execution of tasks inherent to the Didactic Model of Mathematics in Context, linked to Theory Mathematics in the Context of Sciences. From a study on categories of teaching knowledge, in the view of several researchers, we identified those that need to be put into action in the eight tasks that the teacher performs when building a contextualized event, pointing out some of them from an event built for an initial course of Differential and Integral Calculus in a Civil Engineering Program. By means of the analysis carried out, we concluded that most tasks require the mobilization, always in a simultaneous and articulated way, of content, didactic, pedagogical and technological knowledge.
\end{abstract}

Keywords: Categories of teaching knowledge; Didactic Model of Mathematics in the Context of Science; Mathematics; Engineering; Contextualized event.

\section{INTRODUCCIÓN}

Integrando el Grupo de Investigación de Educación Algébrica (GPEA) y los grupos de investigación La Matemática en la Formación Profesional y Educación en Ingeniería, Diseño y Administración, hemos desarrollado investigaciones que se fundamentan en las ideas de la Teoría de la Matemática en el Contexto de las Ciencias (TMCC), referencial construido con el objetivo de apoyar reflexiones acerca de los procesos de enseñanza y aprendizaje de Matemática en cursos universitarios que no pretenden formar matemáticos.

Actualmente, hemos realizado: (i) investigaciones de carácter teórico-bibliográfico y (ii) estudios pretendiendo comprender qué conceptos de la Matemática participan en los diferentes carreras de Ingenierías (en Brasil, existen cerca de 60 carreras de Ingeniería, tales como Química, Eléctrica, Mecánica, de Alimentos etc.), de qué forma se da tal participación y cómo construir, a partir de eso, algo que en la teoría TMCC recibe el nombre de eventos contextualizados, que son problemas o proyectos integrando disciplinas matemáticas y no matemáticas presentes en el programa de estudio de determinado curso universitario.

En el presente artículo, de carácter teórico-bibliográfico, nuestro objetivo es retomar las reflexiones presentadas en Bianchini, Lima y Gomes (2019), ejemplificando, por medio de consideraciones acerca del proceso de construcción y organización didáctica del evento 
contextualizado, expuesto en Gomes et al. (2019), la participación de cada una de las categorías de conocimientos docentes señaladas como esenciales en las tareas inherentes al Modelo Didáctico de la Matemática en Contexto (MoDiMaCo), relacionado a la TMCC. Para poder alcanzar nuestro objetivo, es necesario presentar, en primer lugar, algunas consideraciones al respecto de la TMCC y las diferentes categorías de conocimientos docentes.

\section{TEORIA DE LA MATEMÁTICA EN EL CONTEXTO DE LAS CIENCIAS (TMCC)}

En la Teoría de la Matemática en el Contexto de las Ciencias (TMCC), desarrollada por la investigadora mexicana Patricia Camarena, el proceso educativo es concebido como un sistema complejo en el cual interactúan asuntos curriculares, epistemológicos, didácticos, cognitivos y docentes. Esos asuntos están directamente relacionados a la organización del referencial en cinco fases interdependientes y, por lo tanto, no aisladas (Camarena, 2010; 2013).

En la fase curricular, el objetivo central es reflexionar sobre el currículum de Matemática más adecuado para determinado curso universitario en el cual esa ciencia sirve y efectivamente construir tal currículum. En esta fase, desempeña un papel esencial la metodología Dipcing (Diseño de programas de estudio de matemáticas en carreras de ingeniería), desarrollada por Camarena (2002), que estructura la construcción de programas de Matemática para cursos que no pretenden formar matemáticos, especialmente Ingeniería, a partir de tres vertientes de análisis, cada una proveniente de una de las fases de la metodología.

En la etapa central, se analiza en qué disciplinas no matemáticas de determinado curso universitario se requieren conceptos matemáticos, qué conceptos son esos, si son usados como herramientas o como fundamentos teóricos para la construcción de otros conceptos, con qué nivel de profundidad son usados, qué notaciones se emplean, si estas están o no de acuerdo con las utilizadas en las disciplinas matemáticas.

En la etapa precedente, se realiza una evaluación diagnóstica para comprender qué nivel de conocimientos matemáticos de los que están ingresando en los cursos universitarios en los cuales la Matemática está al servicio para, a partir de ese diagnóstico, analizar qué conceptos de la Educación Básica tienen que ser retomados en el momento en que el estudiante ingresa a la universidad y cuáles podrá estudiar, de manera autónoma, solo con algún tipo de recomendación de material u orientación docente. Finalmente, en la etapa consecuente, se investiga, por medio de entrevistas con profesionales egresados de determinado curso universitario en el cual la Matemática está al servicio, qué conceptos matemáticos se requieren en sus rutinas 
profesionales y si los mismos fueron o no estudiados por ellos en la universidad.

El programa de Matemática para determinado curso se construye entonces a partir de los resultados de los análisis de los datos provenientes de esas tres etapas de la Dipcing y también tomando en consideración la necesidad de que estén presentes en tal programa los conceptos esenciales para mantener la estructura lógica del conocimiento matemático.

En la fase epistemológica de la TMCC, se realizan estudios con el objetivo de comprender de qué manera están vinculados, del punto de vista epistemológico, a determinados conceptos matemáticos, los conceptos específicos de esos cursos universitarios en los cuales la Matemática está al servicio. Tales estudios complementan los realizados en la etapa central de la Dipcing y ayudan al docente a planificar, del punto de vista didáctico, un abordaje interdisciplinario para determinada disciplina matemática y construir los eventos contextualizados.

Esta organización del proceso de enseñanza de acuerdo con los preceptos de la TMCC es el objetivo central en la fase didáctica de tal referencial, que contempla un modelo didáctico denominado MoDiMaCo, en el cual las principales herramientas para la enseñanza de la Matemática son los eventos contextualizados, que, de acuerdo con Camarena (2013), son problemas o proyectos que desempeñan papel de entes integradores entre disciplinas matemáticas y no matemáticas, convirtiéndose en herramientas para el trabajo interdisciplinario en el ambiente de aprendizaje. En el MoDiMaCo, desarrollado a partir de pilares constructivistas, se prevé que el evento sea trabajado de forma colaborativa por los estudiantes, que deben actuar en equipos de tres integrantes, cada uno con un papel: líder emocional, líder intelectual y líder operativo (Camarena, 2017). Para actuar conforme el MoDiMaCo, el docente tiene que desempeñar una serie de tareas (Camarena, 2017; Lima, Bianchini y Gomes, 2018), algunas de las cuales son retomadas en el transcurso de este artículo.

Los efectos, con relación al aprendizaje del estudiante, de una enseñanza de Matemática conducida de acuerdo a los preceptos del MoDiMaCo, son objetos de los análisis realizados en la fase cognitiva de la TMCC, teniendo como base, entre otras, las funciones cognitivas de Feuerstein (1979), la teoría da Aprendizaje Significativo, de Ausubel, Novak y Hanesian (1990), y la de los Campos Conceptuales, de Vergnaud (1990).

Para que los profesores puedan implementar en clase el programa planificado en la fase curricular y conducir sus clases alineadas al MoDiMaCo, inherente a la fase didáctica de la TMCC, es necesario que construyan o profundicen conocimientos relacionados a los contenidos 
que van a enseñar, a los procesos de enseñanza y de aprendizaje, a los cursos universitarios en los que van a actuar y a las tecnologías como mediadoras del aprendizaje. Por esa razón en la fase docente, ganan importancia los programas de formación de profesores para aquellos que enseñan Matemática en cursos en los cuales esa ciencia está al servicio.

A continuación, presentamos consideraciones acerca de las diferentes categorías de conocimientos docentes que, posteriormente, serán articuladas a las tareas desempeñadas por los profesores que actúan según los preceptos del MoDiMaCo.

\section{CATEGORÍAS DE CONOCIMIENTOS DOCENTES}

Basándose en los estudios de Shulman (1986; 1987), Mishra y Koehler (2006) y Ball, Thames y Phelps (2008), los autores Silva y Lima (2015) clasificaron los conocimientos docentes en: de contenido (CC), didáctico (CD), pedagógico $(\mathrm{CP})$ y tecnológico $(\mathrm{CT})$. En la Cuadro 1, presentamos, de manera resumida, a partir de Lima, Bianchini y Gomes (2018), tales categorías de conocimientos y a adaptándolas al caso del profesor que enseña Matemática en cursos que no pretenden formar matemáticos, más específicamente en las Ingenierías. Tal adaptación consiste esencialmente en la inserción de conocimientos sobre la Teoría de la Matemática en el Contexto de las Ciencias y de su modelo didáctico (MoDiMaCo) en el ámbito de CD.

Cuadro1. Categorías de conocimientos docentes.

\begin{tabular}{|c|c|l|}
\hline $\begin{array}{c}\text { Categoría de } \\
\text { Conocimiento }\end{array}$ & Sigla & \multicolumn{1}{c|}{ Descripción } \\
\hline $\begin{array}{c}\text { Conocimiento de } \\
\text { Contenido }\end{array}$ & $\mathrm{CC}$ & $\begin{array}{l}\text { Conocimiento de los contenidos matemáticos - incluyendo sus hechos centrales, } \\
\text { conceptos, teorías, procedimientos - y capacidad de articularlos. }\end{array}$ \\
\hline $\begin{array}{c}\text { Conocimiento } \\
\text { Pedagógico }\end{array}$ & $\mathrm{CP}$ & $\begin{array}{l}\text { Conocimiento genérico de elementos relativos al aprendizaje de los alumnos, } \\
\text { incluyendo, administración de la clase, desarrollo de situaciones de aprendizaje, } \\
\text { importancia de valorar los conocimientos previos de los estudiantes, diferentes } \\
\text { estrategias para evaluar sus comprensiones etc. }\end{array}$ \\
\hline $\begin{array}{c}\text { Conocimiento } \\
\text { Didáctico }\end{array}$ & $\mathrm{CD}$ & $\begin{array}{l}\text { Conocimiento relacionado a cómo enseñar Matemática, en determinada carrera de } \\
\text { ingeniería, a partir de las teorías de la Didáctica de la Matemática, de la Teoría de } \\
\text { la Matemática en el Contexto de las Ciencias y de su modelo didáctico (el } \\
\text { MoDiMaCo). }\end{array}$ \\
\hline
\end{tabular}




\begin{tabular}{|c|c|l|}
\hline $\begin{array}{c}\text { Conocimiento } \\
\text { Tecnológico }\end{array}$ & CT & $\begin{array}{l}\text { Conocimiento relacionado a las habilidades en utilizar tecnologías, como, por } \\
\text { ejemplo, regla, compás y escuadra, y, específicamente, tecnologías más avanzadas, } \\
\text { como Internet, softwares educativos, videos digitales, entre otros. }\end{array}$ \\
\hline
\end{tabular}

\section{Fuente: Elaboración Propia}

Además de las categorías de conocimiento mostradas en la Cuadro 1, Silva y Lima (2015) exhiben otras once categorías obtenidas a partir de intersecciones dos a dos (6), tres a tres (4) y de los cuatro originales. Tales intersecciones se muestran en la Figura 1, construida y presentada originalmente por Silva y Lima (2015).

Figura. 1. Intersecciones entre categorías de conocimientos docentes.

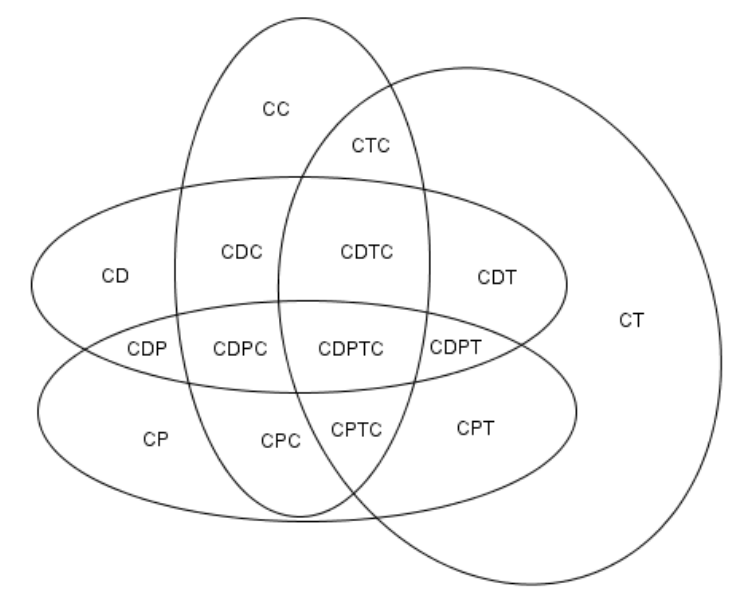

Fuente: Elaborada con base en Silva y Lima (2015).

Cada una de las categorías de conocimientos resultantes de esas intersecciones presentadas en la Figura 1 son brevemente caracterizadas en la Cuadro 2, basado en lo que se presenta en Lima, Bianchini y Gomes (2018).

Cuadro 2. Intersecciones de las categorías de conocimientos docentes.

\begin{tabular}{|c|c|l|}
\hline $\begin{array}{c}\text { Categoría de } \\
\text { Conocimiento }\end{array}$ & Sigla & \multicolumn{1}{c|}{ Descripción } \\
\hline $\begin{array}{c}\text { Conocimiento } \\
\text { Didáctico del } \\
\text { Contenido }\end{array}$ & CDC & $\begin{array}{l}\text { Conocimiento relacionado a la potencialidad de las teorías de la Didáctica de } \\
\text { la Matemática para la enseñanza de determinado contenido y a la capacidad } \\
\text { del profesor de, ante un contenido específico, relacionarlo, por medio del } \\
\text { MoDiMaCo, a los conceptos abordados en las disciplinas específicas y } \\
\text { formación profesional de cada carrera de Ingeniería. }\end{array}$ \\
\hline
\end{tabular}




\begin{tabular}{|c|c|c|}
\hline $\begin{array}{l}\text { Conocimiento } \\
\text { Pedagógico del } \\
\text { Contenido }\end{array}$ & $\mathrm{CPC}$ & $\begin{array}{l}\text { Relación entre el conocimiento de contenidos con los pedagógicos generales, } \\
\text { como, por ejemplo, decidir el mejor método de evaluación para contenidos } \\
\text { específicos o qué contenidos son más adecuados para el trabajo por proyectos. }\end{array}$ \\
\hline $\begin{array}{l}\text { Conocimiento } \\
\text { Tecnológico del } \\
\text { Contenido }\end{array}$ & CTC & $\begin{array}{l}\text { Conocimiento sobre la manera por la cual la tecnología y determinado } \\
\text { contenido están relacionados y también a la capacidad de evaluar, a partir de } \\
\text { la especificidad deseada en la formación del graduando de determinada } \\
\text { carrera de Ingeniería, cuál es el recurso tecnológico más apropiado para } \\
\text { explorar un determinado contenido. }\end{array}$ \\
\hline $\begin{array}{l}\text { Conocimiento } \\
\text { Didáctico } \\
\text { Tecnológico }\end{array}$ & CDT & $\begin{array}{l}\text { Conocimiento que posibilita al docente analizar, tomando en consideración } \\
\text { el MoDiMaCo y las teorías de la Didáctica de la Matemática, de qué manera } \\
\text { pueden ser usadas las diferentes tecnologías en los procesos de enseñanza y } \\
\text { aprendizaje en las diferentes carreras de Ingeniería y cuáles son los recursos } \\
\text { más apropiados para eso. }\end{array}$ \\
\hline $\begin{array}{l}\text { Conocimiento } \\
\text { Pedagógico } \\
\text { Tecnológico }\end{array}$ & $\mathrm{CPT}$ & $\begin{array}{l}\text { Conocimiento sobre cómo las diferentes tecnologías pueden ser usadas en } \\
\text { contextos de enseñanza y aprendizaje y cuáles serán los posibles resultados } \\
\text { de este uso, reflexionando acerca de los cambios introducidos por las } \\
\text { tecnologías en los procesos de construcción de conocimiento, en la } \\
\text { administración de las clases, en las evaluaciones y en la relación entre el } \\
\text { alumno y el saber. }\end{array}$ \\
\hline $\begin{array}{c}\text { Conocimiento } \\
\text { Didáctico Pedagógico }\end{array}$ & CDP & $\begin{array}{l}\text { Conocimiento relacionado al dominio del profesor de utilizar sus } \\
\text { conocimientos pedagógicos generales y los relativos a las teorías de la } \\
\text { Didáctica de la Matemática, para construir, en el ámbito del MoDiMaCo, } \\
\text { situaciones de enseñanza que apunten al aprendizaje de contenidos por parte } \\
\text { de estudiantes de determinada carrera de Ingeniería. }\end{array}$ \\
\hline $\begin{array}{l}\text { Conocimiento } \\
\text { Didáctico Pedagógico } \\
\text { del Contenido }\end{array}$ & CDPC & $\begin{array}{l}\text { Conocimiento que posibilita al profesor reflexionar sobre los aspectos } \\
\text { pedagógicos generales a ser considerados durante los procesos de enseñanza } \\
\text { y aprendizaje, en acuerdo con las teorías de la Didáctica de la Matemática y } \\
\text { el MoDiMaCo, de determinado contenido en una carrera de Ingeniería. }\end{array}$ \\
\hline $\begin{array}{l}\text { Conocimiento } \\
\text { Didáctico } \\
\text { Tecnológico del } \\
\text { Contenido }\end{array}$ & CDTC & $\begin{array}{l}\text { Conocimiento que permite al docente reflexionar, a partir de las teorías de la } \\
\text { Didáctica de la Matemática y del MoDiMaCo, de qué manera, al enseñar un } \\
\text { determinado contenido en una carrera de Ingeniería, se pueden emplear las } \\
\text { diferentes tecnologías. }\end{array}$ \\
\hline $\begin{array}{l}\text { Conocimiento } \\
\text { Didáctico Pedagógico } \\
\text { Tecnológico }\end{array}$ & CDPT & $\begin{array}{l}\text { Conocimiento que permite al profesor analizar, basado en el MoDiMaCo, en } \\
\text { las teorías de la Didáctica de la Matemática y también a partir de sus } \\
\text { conocimientos pedagógicos generales, cómo pueden ser usadas las diferentes } \\
\text { tecnologías en el proceso de enseñanza y aprendizaje, qué resultados puede } \\
\text { generar la introducción de estos recursos para la construcción del }\end{array}$ \\
\hline
\end{tabular}




\begin{tabular}{|c|l|l|}
\hline & & $\begin{array}{l}\text { conocimiento de los estudiantes y, entre las herramientas disponibles, cuáles } \\
\text { son las más adecuadas para ser utilizadas en la enseñanza de Matemática en } \\
\text { cada carrera de Ingeniería. }\end{array}$ \\
\hline $\begin{array}{c}\text { Conocimiento } \\
\text { Pedagógico } \\
\begin{array}{c}\text { Tecnológico del } \\
\text { Contenido }\end{array}\end{array}$ & CPTC & $\begin{array}{l}\text { Conocimiento que permite al profesor, a partir de sus conocimientos } \\
\text { pedagógicos generales, analizar de qué manera pueden ser usadas las } \\
\text { diferentes tecnologías en los procesos de enseñanza y aprendizaje de } \\
\text { determinado contenido, en cada una de las diferentes carreras de Ingeniería y } \\
\text { qué resultados pueden generar la introducción de esos recursos para la } \\
\text { formación de los graduandos. }\end{array}$ \\
\hline $\begin{array}{c}\text { Conocimiento } \\
\text { Didáctico Pedagógico } \\
\text { Tecnológico del } \\
\text { Contenido }\end{array}$ & CDPTC & $\begin{array}{l}\text { Conocimiento que posibilita al profesor buscar, basado en las teorías de la } \\
\text { Didáctica de la Matemática, en el MoDiMaCo, en sus conocimientos } \\
\text { pedagógicos generales, tecnológicos y de contenido, estrategias de enseñanza } \\
\text { adecuadas a cada carrera de Ingeniería, pretendiendo maximizar el } \\
\text { aprendizaje de los estudiantes sobre determinado contenido. }\end{array}$ \\
\hline
\end{tabular}

Fuente: Elaboración Propia

\section{TAREAS PARA CONSTRUIR UN EVENTO CONTEXTUALIZADO: CONOCIMIENTOS MOVILIZADOS}

El profesor que opta por enseñar Matemática en un curso de Ingeniería siguiendo los preceptos del MoDiMaCo tendrá, como se explica en Camarena (2017) e Lima, Bianchini y Gomes (2018), necesariamente, que desempeñar una serie de tareas inherentes a este Modelo Didáctico. Estas, como se presenta en Lima, Bianchini y Gomes (2019), están distribuidas en tres momentos distintos: (i) la construcción del evento contextualizado, (ii) el trabajo en clase con el evento construido y (iii) el trabajo después de aplicar el evento.

En Bianchini, Lima y Gomes (2019), buscamos explicitar las diferentes categorías de conocimientos a ser usadas por los docentes para desempeñar las tareas presentes en cada uno de esos momentos. En esta sección, retomamos la discusión y ejemplificamos, por medio de un evento contextualizado específico, cómo fueron movilizadas, es decir, se pusieron en acción, las diferentes categorías de conocimientos docentes, restringiéndonos, en este trabajo, a las tareas, mostradas en la Cuadro 3, a ser desempeñadas por el profesor en el momento de construir el evento que, según lo que postula la TMCC, se da en el ámbito de la fase epistemológica.

Cuadro 3. Tareas a ser desempeñadas por el docente al construir un evento contextualizado.

\section{Tarea}


1. Identificar situaciones de la Ingeniería a partir de las cuales puedan ser construidos los eventos contextualizados.

2. Analizar las situaciones identificadas para: verificar si en las mismas están presentes los contenidos matemáticos con los cuales se desea trabajar; identificar si los estudiantes poseen los conocimientos previos necesarios para trabajar con el evento a ser propuesto y si tal situación realmente tiene potencial para posibilitar que los graduandos establezcan un vínculo entre los conocimientos previos y los emergentes.

3. Establecer el papel del evento contextualizado que está siendo construido (diagnóstico, motivación, construcción de conocimientos, refuerzo de conocimientos, evaluación, superación de obstáculos etc.).

4. Iniciar la elaboración de la historia del evento contextualizado (descripción del evento, su papel, conocimientos matemáticos y del contexto presentes, conocimientos previos requeridos, posibles maneras de resolución del evento, obstáculos que los alumnos podrán enfrentar, posibles preguntas a ser presentadas por los estudiantes y las respuestas, en forma de preguntas, que podrán ser dadas).

5. Planificar instrumentos (que pueden ser otros eventos con ese papel) para diagnosticar, reforzar y, si fuera el caso, construir, conocimientos previos relacionados al tema a ser trabajado o para identificar y entonces superar posibles obstáculos que pueden ser enfrentados por los estudiantes.

6. Planificar posibles actividades de aprendizaje a ser trabajadas, si fuera el caso con la ayuda de herramientas tecnológicas, si el docente percibe que, durante la resolución del evento, los estudiantes presentan dudas y no logran avanzar más sin su intervención.

7. Planificar las actividades de aprendizaje pretendiendo la descontextualización del contenido trabajado.

8. Planificar cómo hará la evaluación de los aprendizajes y construir las actividades o instrumentos a ser utilizados con esa finalidad.

Fuente: Elaboración Propia

El evento contextualizado utilizado como ejemplo en este artículo, teniendo como público-objetivo estudiantes de una disciplina inicial de Cálculo Diferencial e Integral (CDI), impartida en el curso de Ingeniería Civil, fue construido a partir de un problema clásico del área (para más informaciones consultar Gomes et al. 2018 y Gomes et al. 2019), y que es modelado por una ecuación diferencial de $2^{\mathrm{a}}$ orden, contenido que, en una primera disciplina de CDI, todavía no es accesible para el estudiante. De la misma manera, hay una serie de conceptos específicos de la Ingeniería que tampoco serán, en este momento, de dominio del graduando. Fue necesario, por lo tanto, realizar una adaptación del problema, tanto del punto de vista de la Matemática como de la Ingeniería, para que se tornase viable de ser trabajado con el público pretendido. En la propuesta que presentamos, el estudiante no resolverá la ecuación diferencial involucrada en la situación. Partiendo de la solución que suministraremos, explorará, de manera contextualizada, diferentes contenidos matemáticos que usualmente son objetos de estudio en 
una disciplina inicial de CDI.

Considere un pórtico con masa $m=384 \mathrm{~kg}$, rigidez de cada pilar $\frac{k}{2}=19200 \mathrm{~N} / \mathrm{m}$ y tasa de amortecimiento $\xi=\frac{c}{2 m \sqrt{\frac{k}{m}}}=0,05$, siendo $c$ la constante de amortecimiento. Una fuerza estática es aplicada sobre esa estructura, causando un desplazamiento inicial $u_{0}=0,1 \mathrm{~m}$. En seguida, esa fuerza es retirada bruscamente y la estructura pasa a vibrar libremente con velocidad inicial nula. Considerando solo la posibilidad de desplazamiento horizontal de ese pórtico, la expresión que permite analizar el comportamiento del desplazamiento $u$ en función del tiempo es dada por: $u(t)=e^{-0,5 t}[0,10 \cos (10 t-0,05)]$

¿Cuántos ciclos serán necesarios para que la amplitud de movimiento sea $20 \%$ de la amplitud original?

Como presentamos en Bianchini, Lima y Gomes (2019), para construir un evento contextualizado, el docente tiene que ejecutar ocho tareas, siendo que, para cada una, movilizará diferentes conocimientos. En las Cuadros que presentamos a continuación, detallamos cómo percibimos la movilización de las categorías de conocimiento anteriormente citadas, en el momento en que el profesor ejecuta las ocho tareas inherentes a la construcción de un evento contextualizado.

Cuadro 4. Conocimientos de contenido para las tareas presentes en la construcción de un evento contextualizado.

\begin{tabular}{|c|l|c|}
\hline $\begin{array}{c}\text { Categoría de } \\
\text { Conocimiento }\end{array}$ & \multicolumn{1}{|c|}{ Conocimiento } & Tarea(s) \\
\hline \multirow{5}{*}{ Contenido } & $\begin{array}{l}\text { Conocimiento matemático profundizado de los contenidos desarrollados en } \\
\text { las disciplinas de Matemática presentes en el programa de la carrera de } \\
\text { Ingeniería para el cual se desea construir un evento contextualizado. }\end{array}$ & 1 \\
\cline { 2 - 4 } & $\begin{array}{l}\text { Profundizado conocimiento de los contenidos matemáticos presentes en el } \\
\text { evento contextualizado en cuestión, de cómo estos están articulados entre sí, }\end{array}$ & \multirow{2}{*}{$2,3,4,5}$, \\
& $\begin{array}{l}\text { con aquellos anteriormente trabajados y con los cuales todavía serán } \\
\text { abordados. }\end{array}$ & $6,7,8$ \\
\cline { 2 - 4 } & $\begin{array}{l}\text { Conocimiento, aunque superficial (con la autonomía de buscar profundizar } \\
\text { junto a docentes del área específica) de los contenidos de la Ingeniería y de } \\
\end{array}$ & 4,6 \\
\hline
\end{tabular}

Fuente: Elaboración Propia

Cuadro 5. Conocimientos didácticos para las tareas presentes en la construcción de un evento contextualizado.

\begin{tabular}{|c|l|c|}
\hline $\begin{array}{c}\text { Categoría de } \\
\text { Conocimiento }\end{array}$ & \multicolumn{1}{|c|}{ Conocimiento } & Tarea $(s)$ \\
\hline \multirow{5}{*}{ Didáctico } & $\begin{array}{l}\text { Conocimiento de la fase didáctica de la TMCC y, consecuentemente, de los } \\
\text { aspectos inherentes al MoDiMaCo, en especial de la noción de evento } \\
\text { contextualizado y a las vinculadas a la misma. }\end{array}$ & $1,2,4,5$, \\
\cline { 2 - 3 } & $\begin{array}{l}\text { Conocimiento de la fase curricular de la TMCC y especialmente de la fase } \\
\text { central de la metodología Dipcing, por medio de la cual se establece el } \\
\text { procedimiento a ser adoptado para identificar situaciones con potencial para } \\
\text { generar eventos contextualizados. }\end{array}$ & $1,2,8$ \\
\hline
\end{tabular}




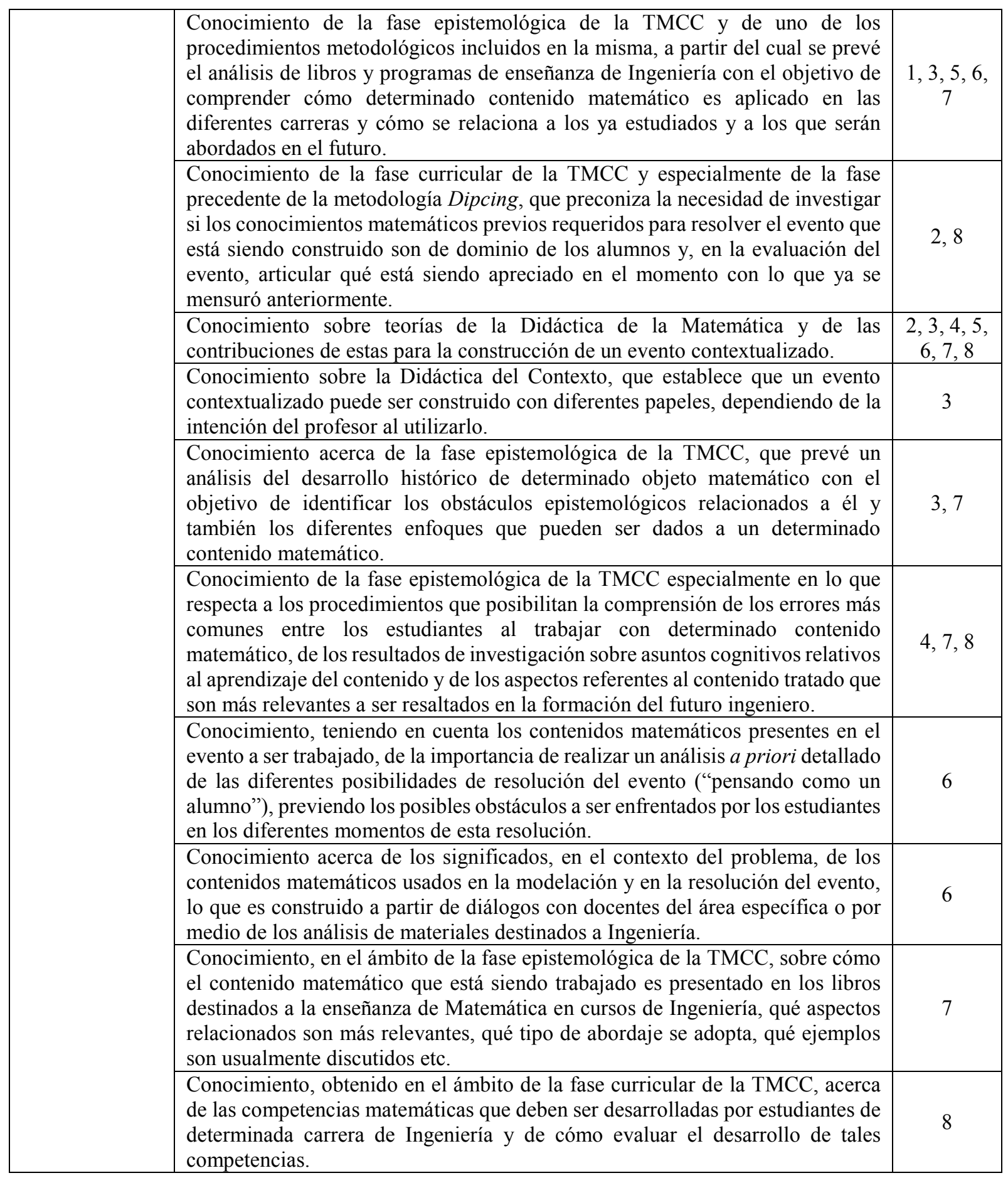

Fuente: Elaboración Propia

Cuadro 6. Conocimientos pedagógicos para las tareas presentes en la construcción de un evento contextualizado.

\begin{tabular}{|c|c|c|}
\hline $\begin{array}{c}\text { Categoría de } \\
\text { Conocimiento }\end{array}$ & Conocimiento & Tarea $(s)$ \\
\hline
\end{tabular}




\begin{tabular}{|c|c|c|}
\hline \multirow{10}{*}{ Pedagógico } & $\begin{array}{l}\text { Conocimiento sobre la importancia de tomar en consideración, en la } \\
\text { construcción, organización didáctica, redacción de la historia y evaluación del } \\
\text { evento contextualizado, los conocimientos previos de los estudiantes para que } \\
\text { ellos puedan efectivamente involucrarse en la resolución del evento y construir } \\
\text { nuevos conocimientos, de forma articulada, a partir de los ya existentes en sus } \\
\text { estructuras mentales. }\end{array}$ & $\begin{array}{l}2,3,4,5 \\
\quad 6,7,8\end{array}$ \\
\hline & $\begin{array}{l}\text { Tener consciencia de que una misma situación de enseñanza puede, en función } \\
\text { de la manera como es organizada, asumir diferentes papeles para el proceso de } \\
\text { aprendizaje del estudiante. }\end{array}$ & 3 \\
\hline & $\begin{array}{l}\text { Conocimiento sobre los diferentes papeles que pueden ser asumidos por el } \\
\text { profesor en el proceso de enseñanza (aquel que institucionaliza el conocimiento, } \\
\text { mediador, que acompaña y realiza la gestión de los aprendizajes etc.), en qué } \\
\text { momentos asumir cada uno de esos papeles y cómo organizar intervenciones } \\
\text { didácticas acordes. }\end{array}$ & 4 \\
\hline & $\begin{array}{l}\text { Conocimientos sobre diferentes instrumentos de enseñanza (secuencias } \\
\text { didácticas, trayectorias de aprendizaje, otros eventos contextualizados, guías de } \\
\text { estudios autodirigidos etc.) y cómo seleccionar lo más adecuado para } \\
\text { determinado objetivo. }\end{array}$ & $5,6,7$ \\
\hline & $\begin{array}{l}\text { Conocimiento acerca de cómo formular preguntas inductoras con el objetivo de } \\
\text { orientar el trabajo de los estudiantes, pero no darles respuestas prontas. }\end{array}$ & 6 \\
\hline & $\begin{array}{l}\text { Conocimiento sobre diferentes estrategias de aprendizaje (por ejemplo, las } \\
\text { metodologías activas) y de los preceptos teórico-metodológicos que las } \\
\text { fundamentan. }\end{array}$ & 7 \\
\hline & $\begin{array}{l}\text { Conocimiento acerca de las potencialidades de las interacciones, en situaciones } \\
\text { de enseñanza y aprendizaje, entre los estudiantes y entre ellos y sus profesores. }\end{array}$ & 7 \\
\hline & $\begin{array}{l}\text { Conocimientos sobre diferentes instrumentos de evaluación (cuestionarios, } \\
\text { ejercicios, elaboración de mapas conceptuales, elaboración de una síntesis sobre } \\
\text { el tema estudiado, realización de un trabajo sobre la historia de los conceptos } \\
\text { etc.). }\end{array}$ & 8 \\
\hline & $\begin{array}{l}\text { Conocimiento sobre las modalidades de evaluación, vinculadas a sus diferentes } \\
\text { papeles, a saber, diagnóstica, formativa y sumativa. }\end{array}$ & 8 \\
\hline & $\begin{array}{l}\text { Conocimiento acerca de cómo evaluar no solamente conocimientos, sino } \\
\text { desarrollo integral del estudiante, contemplando también habilidades y actitudes, } \\
\text { su progreso y su disposición para trabajos en equipos, sus compromisos con las } \\
\text { tareas solicitadas etc. }\end{array}$ & 8 \\
\hline
\end{tabular}

\section{Fuente: Elaboración Propia}

Cuadro 7. Conocimientos tecnológicos para las tareas presentes en la construcción de un evento 
contextualizado.

\begin{tabular}{|l|l|c|}
\hline $\begin{array}{c}\text { Categoría de } \\
\text { Conocimiento }\end{array}$ & \multicolumn{1}{|c|}{ Conocimiento } & Tarea(s) \\
\hline \multirow{7}{*}{ Tecnológico } & $\begin{array}{l}\text { Conocimiento sobre Tecnologías Digitales de Información y Comunicación } \\
\text { (TDIC) disponibles y que pueden ayudar en el proceso de resolución del evento } \\
\text { contextualizado, en la posterior descontextualización del contenido trabajado y en } \\
\text { la evaluación del proceso de aprendizaje. }\end{array}$ & $4,6,7$, \\
& $\begin{array}{l}\text { Conocimiento sobre Ambientes Virtuales de Aprendizaje (AVA) que pueden ser } \\
\text { utilizados como loci auxiliares para la realización de actividades relacionadas a la } \\
\text { resolución del evento y a la descontextualización del contenido. }\end{array}$ & 4,7 \\
\cline { 2 - 4 } & $\begin{array}{l}\text { Conocimiento sobre diferentes objetos de aprendizaje, softwares y applets que } \\
\text { pueden contribuir con los procesos de enseñanza y de aprendizaje de esos } \\
\text { para la descontextualización del contenido trabajado. }\end{array}$ & $4,5,6$, \\
\cline { 2 - 5 } & $\begin{array}{l}\text { Conocimiento acerca de los diferentes AVA que pueden ser utilizados para } \\
\text { realizar evaluaciones diagnósticas de conocimientos previos, para la creación de } \\
\text { caminos de aprendizaje para construir o reforzar los conocimientos previos } \\
\text { cuando no fueran de dominio de los estudiantes, para el desarrollo de determinada } \\
\text { actividad de aprendizaje, para el trabajo descontextualizado con determinado } \\
\text { contenido o para la evaluación del proceso de aprendizaje. }\end{array}$ & $5,7,8$ \\
\cline { 2 - 4 } & $\begin{array}{l}\text { Conocimiento sobre qué Tecnologías son más adecuadas para ayuda en la } \\
\text { elaboración y el desarrollo de determinada actividad de aprendizaje. }\end{array}$ & 6 \\
\hline
\end{tabular}

\section{Fuente: Elaboración Propia}

Para ilustrar la movilización de diferentes categorías de conocimientos evidenciadas en las Cuadros 4, 5, 6 y 7, identificamos a continuación algunos de los conocimientos requeridos en cada una de las ocho tareas, en el caso específico del evento contextualizado presentado.

En la tarea 1, los conocimientos de contenido están relacionados al área de CDI presentes en el programa de Ingeniería Civil. Con relación a los conocimientos didácticos, para identificar la situación que posteriormente sirvió de punto de partida para la construcción del evento contextualizado, asumimos el MoDiMaCo como premisa, de la misma forma que colocamos en acción la etapa central de la metodología Dipcing e uno de los procedimientos metodológicos de la fase epistemológica analizando un libro didáctico (intitulado Lições em mecânica das estruturas: dinâmica, de autoría de Mazzilli et al. y publicado en 2016), señalado por ingenieras civiles que detectaron en tal material situaciones-problema con potencial de generar eventos contextualizados. 
En la tarea 2, con relación a los conocimientos de contenidos matemáticos, los conceptos o ideas que están presentes en la modelación y resolución de la situación original, todavía sin ningún tipo de adaptación, son los siguientes: transformaciones geométricas: simetría y rotación; modelación matemática y la importancia de las funciones en ese proceso; función afín; aplicaciones de funciones exponenciales, trigonométricas y sus inversas y funciones trigonométricas hiperbólicas; análisis e interpretación de representaciones gráficas de funciones; límite de función real de una variable real; teorema del encaje (del sándwich) para limitar una función real de una variable real; tasa de variación relativa; derivada como tasa de variación instantánea; nociones básicas sobre números complejos; resolución de ecuaciones diferenciales de $2^{\circ}$ grado homogéneas y de coeficientes constantes. Sin embargo, como el público-objetivo seleccionado para trabajar con el evento está compuesto por estudiantes de una disciplina inicial de CDI, fue necesaria, a partir de la movilización de nuestros conocimientos pedagógicos relativos a la importancia de considerar los conocimientos previos de los estudiantes, una adaptación de la situación original, teniendo en cuenta los conocimientos matemáticos, físicos y específicos de la Ingeniería Civil que eran de dominio de los estudiantes en ese momento de la graduación ( $1^{\mathrm{er}}$ año). Después de esa adaptación, limitamos los conocimientos de contenido a los siguientes tópicos: función, función exponencial y sus características, función trigonométrica y sus características, representaciones gráficas de funciones, producto de funciones, análisis del comportamiento de funciones a partir de sus representaciones gráficas y concepto intuitivo de límite en el infinito.

Del punto de vista didáctico, destacamos nuestra percepción, como docentes, a partir del conocimiento de la teoría de los Registros de Representación Semiótica de Raymond Duval, de la potencialidad de la situación para la realización de tratamientos y conversiones entre diferentes representaciones del objeto matemático función (Duval, 2003).

Sobre la tarea 3, establecemos como papel para el evento desarrollado rever asuntos relativos al estudio de las funciones trigonométricas y exponenciales reales de una variable real, con un enfoque dirigido a la enseñanza superior y a las aplicaciones con las cuales el estudiante encontrará en las disciplinas específicas de Ingeniería Civil y en su futura actuación profesional. Los conocimientos de contenido requeridos están relacionados a las funciones trigonométricas, exponenciales y sus principales características y de cómo estos objetos matemáticos están relacionados a otros ya conocidos por los alumnos y aquellos que estudiarían posteriormente en 
la disciplina inicial de CDI. Del punto de vista didáctico, los conocimientos docentes requeridos están relacionados al MoDiMaCo y a la comprensión de cómo, en la situación seleccionada para construir el evento, están articulados, del punto de vista epistemológico, los conceptos matemáticos ya mencionados y los específicos de la Ingeniería Civil (la noción de pórtico) y de la Física (tasa de amortecimiento, fuerza estática, rigidez de una estructura, movimiento harmónico simple, movimiento harmónico amortecido). Del punto de vista pedagógico, como docentes teníamos claro que, posiblemente, los estudiantes dominarían los conocimientos matemáticos básicos para resolver el problema. Con relación a los de la Física, aunque algunos ya tendrían que haber sido estudiados en la Educación Básica, hay profundizaciones y vínculos a la idea de pórtico que son necesarios para resolver el problema y, por lo tanto, para los de la Ingeniería Civil, teníamos consciencia que sería necesario prever algún tipo de intervención para que los alumnos pudiesen construirlos o profundizarlos.

La tarea 4 tiene un perfil reflexivo y las acciones de planificación que la misma posibilita fundamentan la realización de las otras. Los conocimientos relativos a los contenidos matemáticos que deben ser de dominio del profesor al trabajar con el evento contextualizado son los mismos ya demandados en las tareas anteriores. Al respecto de los conocimientos relacionados a la Ingeniería Civil y a la Física, como docentes, de manera autónoma, buscamos construirlos, aunque de forma no a fondo, pero pretendiendo suplir las necesidades para dirigir la resolución del evento satisfactoriamente, a partir del establecimiento de una relación dialéctica y cooperativa con físicos e ingenieros civiles. En esta planificación notamos la necesidad de la cooperación de esos profesionales para la preparación de materiales demandada por la tarea subsecuente.

Del punto de vista didáctico, como docentes responsables por la redacción de la historia del evento contextualizado que construimos, destacamos los conocimientos relacionados a la importancia de realizar un análisis detallado a priori, que específicamente consideramos según los preceptos de la Ingeniería Didáctica (Artigue, 1998), del problema a ser resuelto. En este análisis, además de detenernos de manera más amplia, a los aspectos establecidos en la Ingeniería Didáctica, como, por ejemplo, el objetivo del problema, los conocimientos previos de los estudiantes, tanto del punto de vista matemático como de la Ingeniería, las diferentes posibilidades de resoluciones y los posibles obstáculos a ser enfrentados por los alumnos, previmos algunas preguntas que podrían ser hechas por los estudiantes en el momento de la 
implementación del evento y también qué tipos de respuestas les daríamos si surgiesen esas preguntas.

En términos de conocimientos pedagógicos, los mismos fueron accionados en el sentido de planificar los papeles que como docentes podríamos asumir durante diferentes momentos de la resolución del evento. Inicialmente previmos, en la fase de preparación previa de los estudiantes, actuar como creadores de materiales que servirían de base para que el los comprendiesen el contexto en el cual el evento está incluido (sobre tales materiales damos más detalles al comentar la tarea 5, ya que explicitamos los instrumentos elaborados para alcanzar los objetivos). Después, en el momento de resolución del evento, planificamos actuar como mediadores proponiendo a los alumnos una serie de preguntas auxiliares con el objetivo de estimular discusiones que indirectamente les permitirán resolver el problema (tales preguntas también serán evidenciadas en los comentarios acerca de la tarea subsecuente). Finalmente, al terminar el trabajo con el evento, actuaríamos como docentes que institucionalizan el conocimiento con la actividad realizada.

En el caso de los conocimientos tecnológicos que tienen que ser puestos en acción, estuvieron relacionados a las herramientas del AVA que planificamos utilizar y al software GeoGebra. Para más detalles sobre la historia del evento contextualizado, consultar Gomes et al. (2019).

En la tarea 5, con relación a los conocimientos de contenido, esos ya fueron explicitados en las tareas anteriores. En términos de conocimientos didácticos, pedagógicos y tecnológicos referentes a la importancia de considerar los conocimientos previos de los estudiantes, los empleamos al elaborar, junto con físicos e ingenieros civiles, en un lenguaje adecuado, accesible para los estudiantes del $1^{\mathrm{er}}$ año de la Ingeniería, de forma vinculada a lo que habían trabajado en Educación Secundaria y recurriendo a un software que permite simulacros acerca de movimientos harmónicos, textos y videos, ofrecidos en un AVA, destinados a ayudar con la inserción de los alumnos en el contexto del problema, o sea, en la construcción de conocimientos previos relativos a Ingeniería Civil y Física.

Nuevamente conocimientos didácticos, pedagógicos y tecnológicos fueron puestos en acción al planificar preguntas y actividades a ser propuestas a los estudiantes con el objetivo de revisitar o construir conocimientos previos referentes a Matemática, esenciales para resolver el 
evento. Algunos ejemplos de preguntas propuestas (discutidas en detalle en Lima et al., 2020) fueron:

Pregunta 1: ¿La relación $u$ presente en el enunciado del problema remite a algún(os) concepto(s) matemático(s) que ya ha estudiado? ¿Cuál(es)?

Objetivo: realizar un sondeo para saber qué conceptos matemáticos el estudiante será remetido al analizar la expresión algébrica de u y al final llevar al estudiante a percibir que la relación entre el desplazamiento horizontal $u$ del pórtico y el tiempo $t$ es una función.

Pregunta 2: ¿Cuáles son los conjuntos dominio, codominio e imagen de la función $u$, considerando exclusivamente el contexto matemático? ¿Y si consideramos el contexto del problema?

Pregunta 3: Con la ayuda de un software, represente gráficamente la función u y a partir de esa representación gráfica confirme lo que observó en la pregunta 2.

Objetivo: las preguntas 2 y 3 tienen por objetivo posibilitar que el estudiante revea, en el contexto del problema propuesto, tópicos ya estudiados en la Educación Básica.

Pregunta 4: En la expresión algébrica de $u$, ¿identifica la presencia de funciones elementares? ¿Cuáles?

Objetivo: desencadenar, de manera dirigida a sus empleos en la Ingeniería y privilegiando un abordaje computacional, ver de nuevo las nociones relacionadas a las funciones elementares tomando por base la función u presente en el problema del pórtico.

Pregunta 5: La función u fue obtenida a partir de operaciones y/o transformaciones involucrando funciones elementares. Identifique esas funciones, operaciones $y / o$ transformaciones.

Objetivo: desencadenar, a partir de la función $u$ presente en el problema del pórtico, el abordaje de operaciones y transformaciones con funciones elementares. 
Con relación a la tarea 6 , la movilización, de forma articulada, de los conocimientos explicitados por medio de las Cuadros 4, 5, 6, y 7, ocurrió durante el proceso de desarrollo de las once preguntas que presentamos a continuación. Las mismas fueron planificadas con el objetivo de inducir el trabajo de los estudiantes en busca de la solución del evento, pero posibilitándoles desarrollar sus propias estrategias de resolución, actuando como protagonistas en sus aprendizajes. Conviene resaltar que, para elaborar tales preguntas, consideramos, especialmente, conocimientos didácticos relacionados a la teoría de los Registros de Representación Semiótica y conocimientos tecnológicos referentes a las potencialidades del software GeoGebra, para un abordaje dinámico del objeto matemático función.

1. Busquen, rápidamente, cómo se llegó a la expresión que modela el desplazamiento horizontal del pórtico con relación al tiempo.

2. Representen, en el mismo sistema cartesiano, gráficamente, las funciones cuyas expresiones algébricas son dadas por $f(t)=0,10 e^{-0,50 t}$ y $g(t)=\cos (10 t-0,05)$. Intenten hacer un esbozo de los gráficos y después constrúyanlos con GeoGebra.

3. ¿Cuál sería la respuesta del problema si la función u no fuese la del enunciado del problema, sino $u(t)=g(t)=\cos (10 t-0,05)$ ?

4. ¿Cómo podrían obtener la función $\mathrm{u}$ a partir de las funciones $\mathrm{f} y \mathrm{~g}$ ? Haga un bosquejo sin utilizar GeoGebra.

5. A partir de lo que observen en la pregunta 4 , ¿tiene sentido la expresión que modela el comportamiento del desplazamiento $\mathrm{u}$ en función del tiempo ser dada por: $u(t)=$ $e^{-0,50 t}[0,10 \cos (10 t-0,05)]$ ? Justifique su respuesta.

6. Utilizando GeoGebra, representen gráficamente las funciones u y $g$ en un mismo sistema cartesiano. Comparen las representaciones gráficas de u y de $g$, buscando similitudes y diferencias entre los comportamientos de tales funciones.

7. ¿Cuál es el comportamiento de u cuando los valores de t tienden al infinito?

8. Construya, con la ayuda de GeoGebra, en un mismo sistema cartesiano, las representaciones gráficas de $\mathrm{u}, f \mathrm{y}-\mathrm{f}$. ¿Existen valores de $t$ para los cuales $\mathrm{u}(\mathrm{t})=\mathrm{f}(\mathrm{t})$ ? ¿Y valores de $t$ para los cuales $\mathrm{u}(\mathrm{t})=-\mathrm{f}(\mathrm{t})$ ?

9. ¿Cuál es el significado, en el contexto del problema, para $u(t)<0, u(t)=0$ y $u(t)>$ 0 ? 
10. ¿Cómo identificar los ciclos y las amplitudes de la función u a partir de su representación gráfica?

11. Respondan la pregunta: ¿Cuántos ciclos serán necesario para que la amplitud del movimiento sea $20 \%$ de la amplitud inicial?

En el caso del evento contextualizado que consideramos en este artículo, la tarea 7 todavía no fue realizada, ya que el evento fue trabajado solamente en una experiencia piloto. Pero, en todo caso, como el objetivo es rever, pero de manera diferente de la ya vista en la Educación Básica, de forma vinculada a la Ingeniería, con un abordaje computacional, nociones relacionadas al objeto matemático función, entendemos que el docente puede planificar la descontextualización, movilizando los conocimientos mencionados en las Cuadros 4, 5, 6 y 7, inicialmente por medio de clases expositivas dialogadas, en las cuales el profesor será el protagonista, ya que, en este momento, será el responsable por la actividad que, en el ámbito de la Teoría de las Situaciones Didácticas, Brousseau (1986) denomina institucionalización. Los conceptos de función, especialmente funciones exponenciales y trigonométricas, sus respectivos: dominios, imágenes, codominios, períodos y amplitudes (esas dos nociones en el caso de las funciones trigonométricas), representaciones gráficas, operaciones y transformaciones con esas funciones serán institucionalizados por el profesor. Posteriormente, siempre que sea posible, recurriendo a diferentes recursos tecnológicos y estrategias de aprendizaje, podrá planificar actividades, considerando solamente el contexto matemático u otros contextos que no sea el análisis dinámico de una estructura, relativas a estos contenidos para ser solucionadas, individualmente o en grupo por los estudiantes, en clase o por medio de un AVA.

De la misma forma que enfatizamos sobre la tarea 7, aclaramos que la tarea 8 tampoco fue realizada en la experiencia piloto implementada. Sin embargo, para el evento considerado, entendemos que, a título de consideración general, la evaluación puede darse de manera continua a lo largo de todo el proceso y, para concluir el trabajo realizado, el docente podrá solicitar que los estudiantes produzcan, individualmente, un informe detallado de la actividad realizada, buscando explicitar, además de las fases de resolución del evento, los obstáculos enfrentados, las relaciones que establecieron entre los contenidos matemáticos y aquellos específicos de la Ingeniería Civil presentes en el problema y también sus principales aprendizajes debido a la resolución del evento. 
Resaltamos que, comparado con las tareas 1 a 6 , presentamos un menor detalle sobre la movilización de conocimientos docentes en las tareas 7 y 8 , ya que estas, de nuestro punto de vista, dependen mucho del conocimiento de las características, en términos de conocimientos previos, madurez matemática, habilidades y actitudes del grupo específico de estudiantes con los cuales se trabajará. Claro que ese conocimiento es importante también en las demás tareas, pero, a nuestro entender, especialmente en esas dos, gana un papel de relieve y merece destaque también la importancia de la percepción del profesor acerca de lo que observar durante la efectiva implementación del evento.

Cuadro 8. Síntesis de los conocimientos movilizados en las tareas.

\begin{tabular}{|c|c|c|c|c|c|}
\hline \multirow{2}{*}{ Tarea(s) } & \multicolumn{4}{|c|}{ Conocimiento de Conocimiento } & \multirow{2}{*}{ Análisis Final } \\
\cline { 2 - 5 } & $C C$ & $C D$ & $C P$ & $C T$ & CDC \\
\hline 1 & $\mathrm{X}$ & $\mathrm{X}$ & & & CDPC \\
\hline 2,3 & $\mathrm{X}$ & $\mathrm{X}$ & $\mathrm{X}$ & & CDPTC \\
\hline $4,5,6,7$ & $\mathrm{X}$ & $\mathrm{X}$ & $\mathrm{X}$ & $\mathrm{X}$ & $\mathrm{CDP}(\mathrm{T}) \mathrm{C}$ \\
\hline 8 & $\mathrm{X}$ & $\mathrm{X}$ & $\mathrm{X}$ & $(\mathrm{X})$ & $\mathrm{C}$ \\
\hline
\end{tabular}

Fuente: Elaboración Propia

Por intermedio de la Cuadro 8, observamos que, para la construcción de un evento contextualizado, la mayoría de las tareas a ser ejecutadas por el profesor requiere la movilización simultánea y articulada de diferentes categorías de conocimientos docentes. Al menos dos aparecen en las ocho tareas: conocimientos de contenido y didácticos. Cuatro tareas requieren la articulación de todas las categorías de conocimientos explicitadas. Una (tarea 8) presupone la movilización de tres categorías, pero puede también contemplar la cuarta, ya que, a nuestro entender, el profesor debe, en la medida de lo posible, tomar en cuenta, en la planificación de la evaluación, las potencialidades de los recursos tecnológicos para evaluar, de diferentes maneras, aquello que el estudiante pueda haber aprendido por medio del evento contextualizado trabajado.

\section{Consideraciones finales}

En las investigaciones de carácter teórico-bibliográfico que hemos realizado, una de las temáticas abordadas tiene que ver con las competencias a ser desarrolladas por los futuros ingenieros a lo largo del curso, siendo necesario destacar que adoptamos la concepción de competencia propuesta por Camarena (2011), en concordancia con las presunciones de la 
TMCC. Para la autora, posibilitar al graduando en Ingeniería que desarrolle competencias, significa permitir que, como futuro profesional, construya cimientos "para enfrentar una situación-problema haciendo uso de la integración de todo su bagaje de conocimientos, habilidades, actitudes y valores que son movilizados en sus estructuras cognitivas" (Camarena, 2011 como citado en Bianchini et al., 2017, p. 69).

Entendemos que, para proporcionar a los estudiantes el desarrollo de competencias, es necesario que el docente reformule su práctica centrándose en este objetivo. Entra en juego, por lo tanto, el tema de la formación del profesor que enseña Matemática en cursos de Ingeniería. Sobre eso, hemos reflexionado especialmente, como profundizamos en este artículo, acerca de los conocimientos que deben ser movilizados - y, por lo tanto, construidos - por los docentes que optan por reorientar sus prácticas de forma de actuar según preconiza el modelo didáctico intrínseco a la TMCC, denominado Modelo Didáctico de la Matemática en Contexto (MoDiMaCo). Cabe señalar que existen diferentes marcos teóricos sobre el conocimiento docente y que, por tanto, análisis similares a los presentados en este artículo a partir de las consideraciones de Lee Shulman, Punya Mishra, Matthew Koehler, Deborah Ball y colaboradores se pueden realizar con subvenciones de otras teorías, como el modelo Mathematics Teacher's Specialized Knowledge - MTSK, de José Carrillo y colaboradores.

En cuanto a la formación del docente que imparte Matemáticas u otras ciencias básicas en cursos de Ingeniería, entendemos que él, cuándo es contratado por una institución para impartir clases en estos cursos, debe experimentar algún tipo de formación para ejercer tal actividad y, en este sentido, el conocimiento de los preceptos del TMCC, del MoDiMaCo y principalmente el trabajo con eventos contextualizados puede contribuir a un enfoque interdisciplinario entre disciplinas básicas y específicas. En el caso de los docentes que ya están trabajando y que no tuvieron la oportunidad de participar en estos cursos de formación, como señalan Lima, Bianchini y Gomes (2018), es pertinente que investigadores, junto con las instituciones, desarrollan estrategias de autoformación que puedan ser compartidas con los docentes. La estructuración de estas estrategias es uno de los objetivos que pretendemos alcanzar cuando buscamos comprender con claridad los conocimientos que el docente debe movilizar para actuar de acuerdo con MoDiMaCo.

Formar un docente apto a orientar su trabajo pretendiendo el desarrollo de las competencias necesarias para el ejercicio profesional del ingeniero, tanto las específicas de la 
Matemática, como las de carácter más general, se ha tornado una necesidad cada vez más urgente ante currículos oficiales para la graduación en Ingeniería fundamentados a partir de la noción de Educación por Competencias. Camarena (2009; 2018) resalta que, en sentido estricto, al hacer referencia a la Educación por Competencias, en general se piensa en programas estructurados por módulos y no por disciplinas. Sin embargo, destaca que trabajar según esta orientación puede ser un cambio muy brusco para la realidad de muchas instituciones, y por esto, en algunas se ha optado por modelos curriculares de transición, en los cuales se mantienen las matrices curriculares organizadas por disciplinas y no por módulos, pero el trabajo docente se reorienta de forma de incorporar aspectos que posibiliten el desarrollo de competencias, priorizando la interdisciplinaridad.

Siendo la interdisciplinaridad el componente de mayor evidencia en el MoDiMaCo, entendemos que, al orientar su práctica a los preceptos de este Modelo, el docente podrá efectivamente contribuir con el desarrollo de competencias por parte de los futuros ingenieros cumpliendo lo que preconizan diferentes directrices para la formación de esos profesionales. Teniendo en cuenta este aspecto, evidenciar, como iniciamos en este artículo, las categorías de conocimientos a ser desarrollados por los profesores que enseñan Matemática en cursos de Ingeniería para que puedan actuar en consonancia con el MoDiMaCo, se torna una tarea relevante en las áreas de la Educación Matemática y especialmente de la Educación en Ingeniería. En nuestras investigaciones futuras, buscaremos realizar un trabajo semejante evidenciando los conocimientos necesarios para ejecutar las tareas vinculadas a los momentos del trabajo en clase con el evento construido y también después de la aplicación del evento.

\section{Referencias}

Artigue, M. (1998) Ingénierie Didactique. Recherches en Didactique des Mathématiques. 9 (3), 281-308.

Ausubel, D. P., Novak, J. D. y Hanesian,H. (1990) Psicología educativa, un punto de vista cognoscitivo. México: Editorial Trillas.

Ball, D. L., Thames,M. H. y Phelps,G. (2008) Content Knowledge for Teaching : What Makes It Special? Journal of Teacher Education. 59, 389-407.

Bianchini, B. L., Lima, G. L., Gomes, E. y Nomura, J. I. (2017) Competências matemáticas: perspectivas da SEFI e da MCC. Educação Matemática Pesquisa, 19 (1), 4979.https://doi.org/10.23925/1983-3156.2017v19i1p49-79

Bianchini, B. L., Lima, G. L., Gomes, E. (2019) Conhecimentos docentes em ação no Modelo Didático da Matemática em Contexto (MoDiMaCo). Em: XV Conferencia Interamericana de Educación Matemática - Comité Interamericano de Educación Matemática, 2019, Anais. Medellín/Colômbia, 1-8. 
Brousseau, G. (1986) Fondements et Méthodes de la Didactique des Mathématiques. Recherches en Didactique des Mathématiques. Grenoble, 7 (2), 33-116.

Camarena, G.P. (2002) Metodología curricular para las ciencias básicas en ingeniería. Revista Innovación Educativa. 2 (10), 22-28, 2 (11) 4-12.

Camarena, G. P. (2009) Reporte de Programa de investigación titulado: Competencias Matemáticas: Marcos Teóricos y Metodologías. Con No. de registro: SIP-IPN: 558. México: Editorial ESIME-IPN.

Camarena, G. P. (2010) Aportaciones de Investigación al Aprendizaje y Enseñanza de la Matemática en Ingeniería. http://www.ai.org.mx/ai/archivos/ingresos/camarenagallardo/dra. patricia_camarena_g allardo.pdf

Camarena, G. P. (2011) Concepción de competencias de las ciencias básicas em el nível universitário. In: Dipp A.J., Macías A. B. (Org.). Competencias y Educación - miradas múltiples de una relación. México: Instituto Universitario Anglo Español A.C e Red Durango de Investigadores Educativos A.C., 88-118.

Camarena, G. P. (2013) A treita anõs de la teoria educativa "Matemática en el Contexto de las Ciencias". Inovación Educativa. $17-44$. http://www.scielo.org.mx/scielo.php?script=sci arttext\&pid=S166526732013000200003

Camarena, G. P. (2017) Didáctica de la matemática en contexto. Educação Matemática Pesquisa, 19(2), 1-26. https://doi.org/10.23925/1983-3156.2017v19i2p1-26

Camarena, G. P. (2018) Formación por competencias en las ciencias básicas de la ingeniería. Revista Brasileira de Ensino de Ciência e Tecnologia. 11(2), 294-320, https://periodicos.utfpr.edu.br/rbect/article/view/8430/pdf

Duval, R. (2003) Registros de Representações Semióticas e Funcionamento Cognitivo da Compreensão. In: Machado S., D., A. (Org.). Aprendizagem em Matemática: Registros de Representação Semiótica. Campinas: Papirus Editora. Cap. 1, 11 - 34.

Feuerstein, R. (1979) The Dynamic Assessment of Retarded Performers: The Learning Potential Assessment Device, Theory, Instruments and Techniques. Jerusalén, Is.: Park Press.

Gomes, E., Lima, G. L., Bianchini, B. L., Rocha, K. B. y Bolelli, P. M. (2018) Análise Dinâmica de Pórticos: uma oportunidade para a construção de um evento contextualizado para o ensino e a aprendizagem de Álgebra Linear. Em: XLVI Congresso Brasileiro de Educação em Engenharia - Associação Brasileira de Educação em Engenharia, 2018, Anais. Salvador/Brasil, 1-10.

Gomes, E., Lima, G. L., Bianchini, B. L., Rocha, K. B. y Bolelli, P. M. (2019) Evento Contextualizado: estudo de um problema da Engenharia Civil para o ensino de Matemática. Em: XV Conferencia Interamericana de Educación Matemática - Comité Interamericano de Educación Matemática, 2019, Anais. Medellín/Colômbia, 1-8.

Lima, G. L., Bianchini, B. L. y Gomes, E. (2018) Conhecimentos docentes e o Modelo Didático da Matemática em Contexto: reflexões iniciais. Educação Matemática Debate, 2(4), 116 135. https://doi.org/10.24116/emd25266136v2n42018a06

Lima, G. L., Bianchini, B. L., Gomes, E. y Schwertl, S. L. (2020) O problema dos pórticos: uma intervenção didática construída para a disciplina de Cálculo Diferencial Integral. Em: XLVIII Congresso Brasileiro de Educação em Engenharia - Associação Brasileira de Educação em Engenharia, 2020, Anais. Evento online, 1-10.

Mazzilli, C. E. N., André, J. C., Bucalem, M. L. y Cifú, S. (2016) Lições em mecânica das estruturas: dinâmica. São Paulo: Blucher. 
Mishra, P. y Koehler,M. J. (2006) Technological Pedagogical Content Knowledge: a gramework for teacher knowledge. Teachers College Record. 108 (6), 1017-1054.

Shulman, L. (1986) Those who understand: knowledge growth in teaching. Educational Researcher. 15 (2), 4-14.

Shulman, L. (1987) Knowlege and Teaching: foundations of the new reform. Harvard Educational Review. 57 (1), 1-22.

Silva, M. J. F. y Lima, G. L. (2015) Conhecimentos desenvolvidos em um curso de licenciatura em Matemática na modalidade a distância. In P. R. Scott \& Ángel Ruíz (Eds.). Educación Matemática en las Américas (Vol. 2, 113-124). República Dominicana: Comité Interamericano de Educación Matemática.

Vergnaud, G. (1990) La teoría de los campos conceptuales. Recherches en Didactique des Mathematiques. 10(2), 133-170.

Autores(as)

Gabriel Loureiro de Lima. Na Pontifícia Universidade Católica de São Paulo é líder do Grupo de Pesquisa A Matemática na Formação Profissional. Participa, também na PUC-SP, do grupo de pesquisa GPEA (Grupo de Pesquisa em Educação Algébrica). Sua investigação de doutorado, intitulada, A disciplina de Cálculo I do curso de Matemática da Universidade de São Paulo: um estudo de seu desenvolvimento, de 1934 a 1994, defendida em 2012, recebeu menção honrosa na categoria Ensino no Prêmio Capes de Teses 2013. Atualmente é Professor do Departamento de Matemática da Pontifícia Universidade Católica de São Paulo (PUC-SP).

É vice coordenador do GT4 - Ensino Superior - da Sociedade Brasileira de Educação

Matemática (SBEM) e desde 2017 é coordenador do Grupo de Trabalho Ciências Básicas e Matemática na Engenharia da Associação Brasileira de Educação em Engenharia (ABENGE).

Eloiza Gomes. Possui graduação em Licenciatura Em Matemática pela Universidade Presbiteriana Mackenzie (1978), graduação em Bacharel Em Matemática pela Universidade Presbiteriana Mackenzie (1979), mestrado em Educação Matemática pela Pontifícia Universidade Católica de São Paulo (1992) e doutorado em Educação Matemática pela

Pontifícia Universidade Católica de São Paulo (2015). Atualmente é professor associado do Instituto Mauá de Tecnologia e participa, na PUC-SP, do grupo de pesquisa GPEA (Grupo de

Pesquisa em Educação Algébrica). Tem como principal área de interesse o ensino e aprendizagem de Matemática no Ensino Superior, principalmente nos cursos de Engenharia.

Barbara Lutaif Bianchini. Possui graduação em Matemática - Licenciatura pela Pontifícia Universidade Católica de São Paulo (1978), graduação em Matemática Bacharelado pela Pontifícia Universidade Católica de São Paulo (1978), graduação em Licenciatura Em Pedagogia pela Universidade de Franca (1988), mestrado em Educação Matemática pela Pontifícia Universidade Católica de São Paulo (1992) e doutorado em Educação (Psicologia da Educação) pela Pontifícia Universidade Católica de São Paulo (2001). Atualmente é professor associado da Pontifícia Universidade Católica de São Paulo. Tem experiência na área de Educação, com ênfase em Educação algébrica, atuando principalmente nos seguintes temas: educação algébrica, educação matemática, álgebra linear, registros de representação semiótica e formação de professores. 